\title{
Icaritin, a novel plant-derived osteoinductive agent, enhances the osteogenic differentiation of human bone marrow- and human adipose tissue-derived mesenchymal stem cells
}

\author{
TAO WU ${ }^{1 *}$, TAO SHU $^{2 *}$, LE KANG $^{3 *}$, JINHUI WU $^{1}$, JIANZHOU XING ${ }^{1}$, \\ ZHIQIN LU ${ }^{1}$, SHUXIANG CHEN ${ }^{3}$ and JUN LV ${ }^{1}$
}

\begin{abstract}
${ }^{1}$ Department of Emergency, Guangdong Provincial Corps Hospital of the Chinese People's Armed Police Forces, Guangzhou Medical University, Guangzhou, Guangdong 510507; ${ }^{2}$ Department of Spine Surgery, The Third Affiliated Hospital of Sun Yat-sen University, Guangzhou, Guangdong 510630;

${ }^{3}$ Department of Orthopaedics, Wuyi TCM Hospital of Jiangmen City, The Sixth Affiliated Hospital of the Medicine College of Jinan University, Jiangmen, Guangdong 529031, P.R. China
\end{abstract}

Received June 1, 2016; Accepted January 19, 2017

DOI: $10.3892 /$ ijmm.2017.2906

\begin{abstract}
For the treatment of diseases affecting bones using bone regenerative medicine, there is an urgent need to develop safe, inexpensive drugs that can strongly induce bone formation. In the present study, we systematically investigated the effects of icaritin, a metabolic product of icariin, on the osteogenic differentiation of human bone marrow-derived mesenchymal stem cells (hBMSCs) and human adipose tissue-derived stem cells (hADSCs) in vitro. After treatment with icaritin at concentrations of $10^{-8}-10^{-5} \mathrm{M}, \mathrm{hBMSC}$ and hADSCs were examined for alkaline phosphatase activity, osteocalcin (OC) secretion, matrix mineralization and expression levels of bone-related mRNA and proteins. Data showed that icaritin at concentrations $10^{-7}-10^{-5} \mathrm{M}$ significantly increased alkaline phosphatase activity, $\mathrm{OC}$ secretion at different time points, and calcium deposition at day 21 . In addition, icaritin upregulated the mRNA expression of genes for bone morphogenetic proteins (BMP-2, -4 and -7), bone transcription factors (Runx2 and Dlx5) and bone matrix proteins (ALP, OC and Col-1). Moreover, icaritin increased the protein levels of BMPs, Runx 2 and OC, as detected by western blot analysis. These findings suggest that icaritin enhances
\end{abstract}

Correspondence to: Dr Jun Lv, Department of Emergency, Guangdong Provincial Corps Hospital of Chinese People's Armed Police Forces, Guangzhou Medical University, 268 Yanling Road, Guangzhou, Guangdong 510507, P.R. China

E-mail: cnwdoc@163.com

*Contributed equally

Key words: icaritin, icariin, bone morphogenetic proteins, osteogenic differentiation, bone marrow-derived mesenchymal stem cells, adipose tissue-derived stem cells the osteogenic differentiation of hBMSCS and hADSCs. Icaritin exerts its potent osteogenic effect possibly by directly stimulating the production of BMPs. Although the osteogenic activity of icaritin in vitro was inferior to that of rhBMP-2, icaritin displayed better results than icariin. Moreover, the low cost, simple extraction procedure, and an abundance of icaritin make it appealing as a bone regenerative medicine.

\section{Introduction}

Bone defects and nonunion caused by trauma, tumor or osteomyelitis present major clinical challenges. These conditions are difficult to treat, and inadequate treatment may handicap patients both financially and medically by prolonging the disability. Local delivery of growth factors and cytokines has been widely used for the enhancement of therapeutic effects in bone regeneration (1-3). Although bone morphogenetic proteins (BMPs) are considered as ideal osteoinductive factors for osteogenic progenitors, the extraction and recombination process of BMPs is complicated; the preparation cost is rather high and it shows low productivity $(4,5)$. In addition, the short biological half-life, weak stability, and demand for optimum controlled-release carriers for therapeutic applications limit their clinical use (6-8). There is evidence that BMPs stimulate osteoclastic activity and lack tissue-selectivity, which can lead to osteoclastic bone resorption $(9,10)$ and ectopic bone formation (11). Therefore, simple, effective, safe and inexpensive bioactive factors need to be developed for treating bone diseases using bone regenerative medicine.

In previous studies, we found that icariin $\left(\mathrm{C}_{33} \mathrm{H}_{40} \mathrm{O}_{15}\right.$; molecular weight, 676.67), a typical flavonol glycoside, is considered to be the major pharmacologically active ingredient of Herba Epimedii and exerts osteoinductive activity. Icariin improved proliferation and osteogenic differentiation of bone marrow-derived mesenchymal stem cells (BMSCs) and promoted bone-defect healing in New Zealand rabbits by combining with scaffolds in vitro (7,12-14). However, recent evidence has shown that icariin is enzymatically hydrolyzed by 
intestinal bacteria and is subsequently metabolized to icaritin $\left(\mathrm{C}_{21} \mathrm{H}_{22} \mathrm{O}_{7}\right.$; molecular weight, 386.4) and desmethylicaritin in vitro $(15,16)$. Notably, the pharmacological effects of icaritin were found to be more potent than those of icariin in vitro $(17,18)$. There is increasing evidence that icaritin possesses estrogen-like activity $(19,20)$, can improve the proliferation and differentiation of osteoblasts and facilitate matrix calcification. In addition, it can suppress the activity of osteoclasts in vitro $(17,21,22)$, which exerts bone-protective function by increasing bone formation and inhibiting bone resorption (23). Further studies have demonstrated that icaritin has the ability to enhance the expression of osteogenic-related mRNA levels in human BMSCs (hBMSCs) (24), and it has already been incorporated into various biomaterials to promote bone repair $(25-28)$. These results indicate that icaritin is a potential osteogenic inductive agent and can be used in bone tissue engineering. Moreover, as icaritin is a small molecule, it has the advantages of being chemically stable, is readily available, does not denature (29), has low cost, and requires simple extraction technology. Thus, icaritin provides a convenient method for preparing drug-loading scaffolds.

The main purpose of this study was to evaluate the effects of icaritin on osteogenic differentiation of hBMSCs and human adipose tissue-derived stem cells (hADSCs) and the expression of bone formation genes and proteins in vitro, and to investigate the feasibility of icaritin to serve as an osteogenic inductive agent.

\section{Materials and methods}

Reagents. Icaritin (>98\% purity) and icariin (>98\% purity) were purchased from Shanghai U-sea Biotech Co. Ltd. (Shanghai, China). Recombinant human BMP-2 (rhBMP-2) was purchased from Peprotech, Inc. (Rocky Hill, NJ, USA). Dulbecco's modified Eagle's medium (DMEM) and fetal bovine serum (FBS) were purchased from HyClone Laboratories (Logan, UT, USA); dimethyl sulfoxide (DMSO), 3-(4,5-dimethylthiazol-2-yl)-2,5-diphenyltetrazolium bromide (MTT), trypsin and TRIzol reagent were purchased from Gibco BRL (Gaithersburg, MD, USA); alkaline phosphatase activity kit and micro-BCA assay kit were obtained from Beyotime Biotech (Jiangsu China). Osteocalcin (OC) ELISA kit was obtained from Biocompare (South San Francisco, CA, USA). Primers were synthesized by Shanghai Yingjun Biotechnology Company (Shanghai, China). All other reagents were of analytical grade. As icaritin and icariin are insoluble in water, they were dissolved in DMSO and used directly in cell culture treatment. The final concentration of DMSO used in the culture was $0.05 \%(\mathrm{v} / \mathrm{v})$.

Cell culture. hBMSCs and hADSCs were obtained from Jennio Biological Technology Co., Ltd. (Guangzhou, China). The cells were routinely maintained in DMEM, supplemented with $10 \% \mathrm{FBS}$ and antibiotics $(50 \mathrm{U} / \mathrm{ml}$ of penicillin and $50 \mathrm{mg} / \mathrm{ml}$ streptomycin; Gibco BRL) at $37^{\circ} \mathrm{C}$ in a humidified atmosphere containing $5 \% \mathrm{CO}_{2}$. The culture media were changed every two days. When the cells reached $80 \%$ confluence, they were digested with $0.25 \%$ trypsin and $0.02 \%$ ethylenediaminetetraacetic acid (EDTA; Amresco, LLC, Solon, OH, USA) and then subcultured at a ratio of 1:3.
Cytotoxicity test of icaritin. hBMSCs and hADSCs were seeded at a density of 5,000 cells/well in a 96-well plate. They were incubated for $24 \mathrm{~h}$ prior to the addition of $150 \mu \mathrm{l}$ icaritin (icaritin doses were $10^{-8}, 10^{-7}, 10^{-6}, 10^{-5}, 10^{-4}$ and $2.0 \times 10^{-4} \mathrm{M}$ separately) media and $0.05 \%$ (v/v) DMSO media, while the control cells were incubated with fresh DMEM and $0.05 \%$ (v/v) DMSO medium. After $48 \mathrm{~h}$, the number of viable cells was detected by MTT assay using a universal microplate spectrophotometer (BioTek Instruments, Inc., Winooski, VT, USA). The cell survival rate $(\%)=\mathrm{OD}_{\text {treatment }} / \mathrm{OD}_{\text {control }} \times 100$.

Alkaline phosphatase (ALP) activity assay and ALP staining. hBMSCs and hADSCs were cultured in 6-well plates at a density of $1 \times 10^{6}$ cells per well. They were treated with $10^{-8}$, $10^{-7}, 10^{-6}$ or $10^{-5} \mathrm{M}$ icaritin (with $0.05 \%$ DMSO), $0.05 \%$ DMSO, $10^{-6} \mathrm{M}$ icariin (with $0.05 \%$ DMSO) or $100 \mathrm{ng} / \mathrm{ml} \mathrm{rhBMP}-2$ (with $0.05 \%$ DMSO) separately. Incubation was carried out for 3, 7 and 11 days. The cells were lysed in $50 \mu \mathrm{l}$ cell lysis buffer. ALP activity and protein content in the cell lysates were determined using an ALP activity kit and a micro-BCA assay kit separately, and the ALP activity was normalized to the corresponding total protein concentration $(\mathrm{U} / \mu \mathrm{g})$. For ALP staining, the cells treated for 11 days were fixed and incubated with $0.5 \mathrm{ml}$ nitroblue tetrazolium (NBT)/ 5-bromo-4-chloro-3-indolyl phosphate solution in the absence of light for $30 \mathrm{~min}$ at room temperature.

$O C$ secretion assay and measurement of mineralized matrix formation. hBMSCs and hADSCs were cultured in 6 -well plates at a density of $1 \times 10^{6}$ cells per well, and they were treated with $10^{-8}, 10^{-7}, 10^{-6}$ or $10^{-5} \mathrm{M}$ icaritin (with $0.05 \%$ DMSO), $0.05 \%$ DMSO, $10^{-6} \mathrm{M}$ icariin (with $0.05 \% \mathrm{DMSO}$ ) or $100 \mathrm{ng} / \mathrm{ml} \mathrm{rhBMP}-2$ (with $0.05 \%$ DMSO) separately. At day 7,14 and 21 , the cells were lysed in $50 \mu 1$ cell lysis buffer, and the OC levels were measured using an ELISA kit. The assay was performed according to the manufacturer's specifications. The absorbance of each well was measured at $450 \mathrm{~nm}$ by an ELISA plate reader. At day 21, the cells were fixed and stained for $1 \mathrm{~min}$ with $0.1 \%$ (w/v) Alizarin Red S. The stained, calcified nodules that appeared bright red in color were photographed.

Reverse transcription-quantitative polymerase chain reaction $(R T-q P C R)$. hBMSCs and hADSCs were cultured in 6-well plates at a density of $1 \times 10^{6}$ cells per well. They were treated with $10^{-6} \mathrm{M}$ icaritin (with $0.05 \%$ DMSO) for 3, 7, 14 and 21 days, and the control cells were treated, respectively, with $0.05 \%$ DMSO, $10^{-6} \mathrm{M}$ icariin (with $0.05 \%$ DMSO) or $100 \mathrm{ng} / \mathrm{ml} \mathrm{rhBMP}-2$ (with $0.05 \%$ DMSO) for the same period. Total cellular RNA was extracted using an RNA extraction kit (Takara Bio, Inc., Otsu, Japan). cDNA was prepared using RNA isolated from these cells (Fermentas, Glen Burnie, MD, USA). Quantitative PCR (qPCR) was performed and monitored using an ABI Prism 7900 Sequence Detection System (Perkin-Elmer Applied Biosystems, Foster City, CA, USA). The housekeeping gene, glyceraldehyde-3-phosphate-dehydrogenase, was used as an endogenous reference gene to normalize the calculation by comparative $\mathrm{Ct}$ (cycle of threshold) value method, and the data were analyzed by $\Delta \Delta \mathrm{C}_{\mathrm{T}}$ relative qualification methods. The data from the icaritin, icariin, and rhBMP-2 groups were 
Table I. Sequences of the primers used for RT-qPCR.

\begin{tabular}{lll}
\hline Gene & \multicolumn{1}{c}{ Forward $\left(5^{\prime} \rightarrow 3^{\prime}\right)$} & \multicolumn{1}{c}{ Reverse $\left(5^{\prime} \rightarrow 3^{\prime}\right)$} \\
\hline Dlx5 & CTCAGAAGGGAAGGAAGGTGATG & ATACCTCACAACCGCCAATCC \\
Runx2 & TGGTTACTGTCATGGCGGGTA & TCTCAGATCGTTGAACCTTGCTA \\
BMP-2 & GCCAGGAGAGCAGGGACG & GTAGTAGAGCCCACAGGCATTG \\
BMP-4 & TTTGCTACAGTCCAAAAGATGAGG & TCATTCCCTCCACAGCCAGTA \\
BMP-7 & AACAAATGTGGCAATTATTTGGATC & ATTTGTCTTAAGTCCTGGTGTTGT \\
ALP & CGGCTTTATCATTCCTTTCCAGTT & CCTGAAATTAAAGACACAATGTGCC \\
OC & GTTACAGTATTCCAGCAGACTCAAAT & CCATACGGTCTTTTGTCACTGTTTT \\
COL-1 & GGAGGATACCCTTGACAAATACTGT & CGACGGGCAAAGCACTCAT \\
GAPDH & CCGAGGGCCCACTAAAGG & GCTGTTGAAGTCACAGGAGACAA
\end{tabular}

expressed as fold-changes relative to the corresponding DMSO control group. The primer sequences of the testing genes are listed in Table I.

Protein extraction and western blot analysis. hBMSCs and hADSCs were seeded in $75-\mathrm{cm}^{2}$ culture flasks (Nunc, Roskilde, Denmark) at a density of $1 \times 10^{7}$ cells per bottle and allowed to attach for $24 \mathrm{~h}$ followed by the addition of $10^{-6} \mathrm{M}$ icaritin (with $0.05 \%$ DMSO). The control cells were incubated in the medium with $0.05 \%$ DMSO, $10^{-6} \mathrm{M}$ icariin (with $0.05 \%$ DMSO) or $100 \mathrm{ng} / \mathrm{ml}$ rhBMP-2 (with $0.05 \%$ DMSO) separately. After incubation for 14 days, the cells were harvested at $4^{\circ} \mathrm{C}$ and lysed in $100 \mathrm{ml}$ of radioimmunoprecipitation assay buffer (Sigma, St. Louis, MO, USA) with $1 \mathrm{mg} / \mathrm{ml}$ aprotinin (Sigma) and protease inhibitor cocktail (Sigma). The lysates were cleared by centrifugation $\left(4^{\circ} \mathrm{C}, 16,000 \mathrm{x} \mathrm{g}, 30 \mathrm{~min}\right)$. The total protein content in each lysate was quantified using a modified Lowry assay (DC protein assay; Bio-Rad Laboratories, Inc., Hercules, CA, USA). The protein samples were fractionated by electrophoresis on a $10 \%$ polyacrylamide gel and transferred to a polyvinylidene difluoride membrane (Pall-Gelman, Port Washington, NY, USA) using a semi-dry blot system. The blots were then blocked in 5\% skim milk in Tris-buffered saline containing Tween-20 (PBS/T) for $1 \mathrm{~h}$ at room temperature. Goat anti-human Runx2 antibody (Cat. no. AF2006; 1:1,000 dilution; R\&D Systems, Inc., Minneapolis, MN, USA) and mouse anti-human OC antibody (Cat. no. H95152M; 1:1,000 dilution; Meridian Life Science, Inc., Memphis, TN, USA) and rabbit anti-human BMP antibody (Cat. no. BA0624; 1:500 dilution; Boster Co., Wuhan, China) were added to $5 \%$ milk, and each blot was incubated overnight at $4^{\circ} \mathrm{C}$. The blots were washed three times for 5 min each with $10 \mathrm{ml}$ TBS/T and incubated with secondary antibody [anti-goat HRP (sc-2354), anti-mouse HRP (sc-2371) or anti-rabbit HRP (sc-2357), 1:3,000 dilution; Santa Cruz Biotechnology, Inc., Dallas, TX, USA] in $10 \mathrm{ml} \mathrm{TBS} / \mathrm{T}$ with gentle agitation for $1 \mathrm{~h}$ at room temperature. The blots were then detected using an electrogenerated chemiluminescence reagent (Kaiji Biotechnology, Nanjing, China) followed by exposure to X-ray film.

Statistical analysis. All experiments were performed in triplicate and representative experiments are shown. Each value was expressed as the mean \pm SD. Statistical comparisons of multiple groups were performed using one-way analysis of variance (ANOVA) with Bonferroni's post hoc test. In all cases, $\mathrm{P}<0.05$ was considered to indicate a statistically significant difference.

\section{Results}

Cytotoxicity of icaritin. The OD values of hBMSCs and hADSCs were stable following treatment with $10^{-8}-10^{-5} \mathrm{M}$ icaritin, indicating that cytotoxicity was undetectable when the concentration of icaritin was less than $10^{-5} \mathrm{M}$ (Fig. 1). However, icaritin demonstrated the ability to decrease cell viability significantly at dosages above $10^{-5} \mathrm{M}(\mathrm{P}<0.01)$, suggesting that high concentrations of icaritin could inhibit cell proliferation. The DMSO group did not show a significant decrease in cell viability, indicating that $0.05 \%(\mathrm{v} / \mathrm{v}) \mathrm{DMSO}$ was safe for the cells and could be used as a co-solvent for icaritin.

Icaritin promotes osteogenic differentiation in hBMSCs and hADSCs. Icaritin showed a time-dependent effect on ALP activity and OC levels in the hBMSC and hADSC cultures. ALP activity increased at day 3 in the hBMSCs and hADSCs in the $10^{-7}-10^{-5} \mathrm{M}$ icaritin group, and it increased significantly with time (Fig. 2A). As ALP is an early marker of osteogenic differentiation, ALP activity assay and ALP staining were used for estimating the effects of icaritin on osteogenic differentiation in cells. In the cells cultured for 11 days, ALP activity in the $10^{-6} \mathrm{M}$ icaritin group was $\sim 107$-fold (hBMSCs) and 101-fold (hADSCs) higher than that in the DMSO group. At day 11, ALP staining reflected the number of hBMSCs and hADSCs that underwent osteogenic differentiation, which is correlated with the levels of ALP activity (Fig. 2B). OC and mineralization are terminal markers of differentiation and maturity of osteoblasts. The treatment of hBMSCs and hADSCs with $10^{-7}-10^{-5} \mathrm{M}$ concentrations of icaritin significantly enhanced the levels of OC at day 14 and 21 compared with the level noted in the DMSO-treated cells $(\mathrm{P}<0.05$; Fig. 2C). After 21 days of treatment, Alizarin Red S staining for mineralization demonstrated that $10^{-7}-10^{-5} \mathrm{M}$ of icaritin could induce the colony growth of hBMSCs and hADSCs and form calcified nodules, and $10^{-6} \mathrm{M}$ icaritin was observed to be the optimum concentration (Fig. 2D). At a different time point, $10^{-6} \mathrm{M}$ icaritin resulted in the highest expression of ALP 


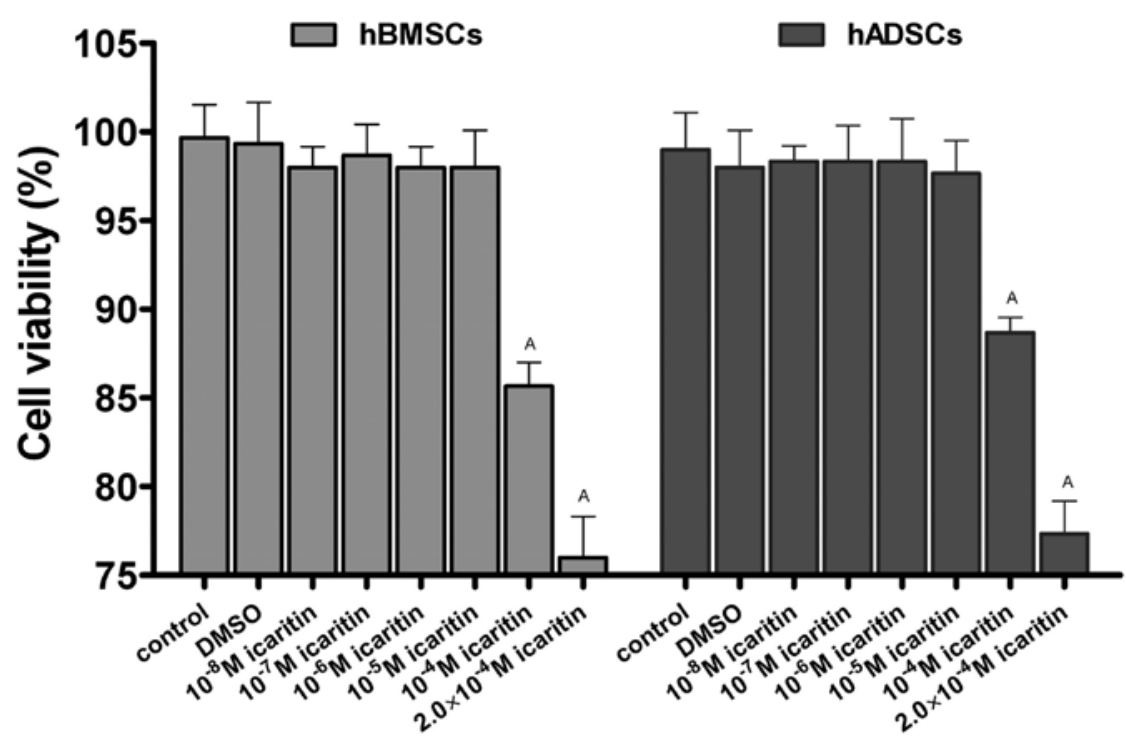

Figure 1. Cytotoxicity of icaritin. Cells were exposed to control, DMSO or $10^{-8}, 10^{-7}, 10^{-6}, 10^{-5}, 10^{-4}, 2.0 \times 10^{-4} \mathrm{M}$ icaritin respectively for $48 \mathrm{~h}$. Cell proliferation was determined by MTT assay. Data are the means $\pm \mathrm{SD}\left(\mathrm{n}=6,{ }^{\mathrm{A}} \mathrm{P}<0.05\right.$ vs. control); $\mathrm{n}$, number of experiments.

and OC in all icaritin groups, and the expression level was higher than that noted in the DMSO and other icariin groups $(\mathrm{P}<0.05)$, but lower than that observed in the rhBMP-2-treated group $(\mathrm{P}<0.05)$. However, icaritin at $10^{-8} \mathrm{M}$ showed no significant effects on the expression of ALP and OC at each time point compared with DMSO $(\mathrm{P}<0.05)$.

Icaritin increases osteogenic mRNA expression. The osteogenic differentiation mechanism of icaritin was assessed using RT-qPCR for marker genes (Fig. 3). The mRNA expression of BMP-4, BMP-7, Dlx5, Runx2, and ALP was significantly upregulated in hBMSCs that were cultured with icaritin $\left(10^{-6} \mathrm{M}\right)$ for 3 days, compared with that in the DMSO control group $(\mathrm{P}<0.05)$. All the osteogenic genes were upregulated by icaritin after 7 days of culture, with the most pronounced changes being observed for Runx2. In addition, the gene expression levels increased with time. After 21 days of culture with icaritin, the mRNA expression of BMP-2, BMP-4, BMP-7 was 6.7-, 11.9-, and 15.5-fold higher than that in the DMSO control, respectively. Moreover, the mRNA expression levels of Dlx 5 and Runx 2 were 4.5- and 120-fold higher than levels in the DMSO control, respectively. The mRNA expression profiles of ALP, COL-1, and OC were 20.7-, 2.6-, and 20.8-fold higher than levels in the DMSO control, respectively. Similarly, for hADSCs, icaritin had significant stimulatory effects on the mRNA expression of BMP-2, Dlx5, Runx2, and ALP genes at day 3 of the culture (vs. DMSO control, $\mathrm{P}<0.05$ ), which is the super-early phase. In addition, at day 7 , all the osteogenic genes were upregulated. The mRNA levels were steadily upregulated by icaritin in a time-dependent manner. After 21 days of culture, BMP-2, -4, -7 , and osteogenic regulatory genes were upregulated by $6.4-$, 14.9- and 14.0-fold when compared with that in the DMSO control, respectively. The osteogenic transcription factor genes, Dlx5 and Runx2, were 5.7- and 130-fold higher than that in the DMSO control, respectively. In addition, the expression levels of bone matrix genes including ALP, Col-1, and OC were
18.3-, 2.5- and 19.0-fold higher, compared with these levels in the DMSO control, respectively. Taken together, the data obtained showed that icaritin, icariin and rhBMP-2 had the ability to promote osteogenic gene expression in hBMSCs and hADSCs. However, there were differences among the three groups in regards to the levels of gene expression at different time points. Although the mRNA expression results of icaritin were inferior to rhBMP-2, icaritin displayed better results than icariin, which served as the second positive control.

Icaritin increases protein expression of BMPs, Cbfal and $O C$ in hBMSCs. The osteogenic differentiation of hBMSCs and hADSCs after treatment with icariin, icaritin or rhBMP-2 for 14 days is represented in Fig. 4. Similar to rhBMP-2, icaritin and icariin increased the expression of BMPs, Runx2 and OC, respectively, while the DMSO control group showed no effects on these proteins. Although OC protein expression following treatment of icaritin in the hBMSCs was not significantly higher than that of the icariin group, the BMP and Runx 2 protein expression levels were significantly higher than levels noted in the icariin groups. In addition, the OC protein expression following treatment with icaritin in the hADSCs was noticeably higher than that noted following treatment with icariin. Protein expression detection using western blot analysis confirmed that the icaritin group showed higher expression of osteogenesis-related proteins compared with levels noted in the icariin group.

\section{Discussion}

Icariin, a type of phytoestrogen, has been used in bone tissue engineering research as a bone induction factor (30,31). After oral administration, icariin is hydrolyzed and deglycosylated by intestinal bacteria. It is then transformed into icaritin and desmethylicaritin, which are responsible for exerting pharmacological activities $(20,32)$. Studies have found that icaritin has a stronger pharmacological activity than icariin $(33,34)$; icaritin 



Figure 2. Effects of icaritin on the osteogenic differentiation in hBMSCs and hADSCs. (A) Time-dependent effects of icaritin on ALP activity in cultured hBMSCs and hADSCs. Data are the means $\pm \mathrm{SD}\left(\mathrm{n}=6,{ }^{\mathrm{A}} \mathrm{P}<0.01\right.$ vs. control, ${ }^{\mathrm{P}} \mathrm{P}<0.05$ vs. control; ${ }^{\mathrm{B}} \mathrm{P}<0.01$ vs. icariin, ${ }^{\mathrm{b}} \mathrm{P}<0.05 \mathrm{vs}$. icariin; ${ }^{\mathrm{C}} \mathrm{P}<0.01$ vs. rhBMP-2, ${ }^{\mathrm{c}} \mathrm{P}<0.05$ vs. rhBMP-2). (B) ALP staining in hBMSCs and hADSCs treated with DMSO, icariin, $10^{-8}, 10^{-7}, 10^{-6}, 10^{-5} \mathrm{M}$ icaritin or rhBMP-2 control for 11 days. The ALP-positive cells appear blue in color. (C) Time-dependent effects of icaritin on osteocalcin (OC) levels in cultured hBMSCs and hADSCs. Data are the means $\pm \mathrm{SD}\left(\mathrm{n}=6,{ }^{\mathrm{A}} \mathrm{P}<0.01\right.$ vs. control, ${ }^{\mathrm{a}} \mathrm{P}<0.05$ vs. control; ${ }^{\mathrm{B}} \mathrm{P}<0.01$ vs. icariin, ${ }^{\mathrm{b}} \mathrm{P}<0.05$ vs. icariin; ${ }^{\mathrm{C}} \mathrm{P}<0.01$ vs. rhBMP- 2 , ${ }^{\mathrm{C}} \mathrm{P}<0.05$ vs. rhBMP-2). (D) Alizarin Red $\mathrm{S}$ staining for mineralization after incubation of the hBMSCs and hADSCs with DMSO control, icariin, $10^{-8}, 10^{-7}, 10^{-6}, 10^{-5} \mathrm{M}$ icaritin or rhBMP-2 control for 21 days. The calcified nodules appear red in color. ALP, akaline phosphatase; hBMSCs, human bone marrow-derived mesenchymal stem cells; hADSCs, human adipose tissue-derived stem cells; rhBMPs, recombinant human bone morphogenetic proteins; DMSO, dimethyl sulfoxide; SD, standard deviation.; $n$, number of experiments 

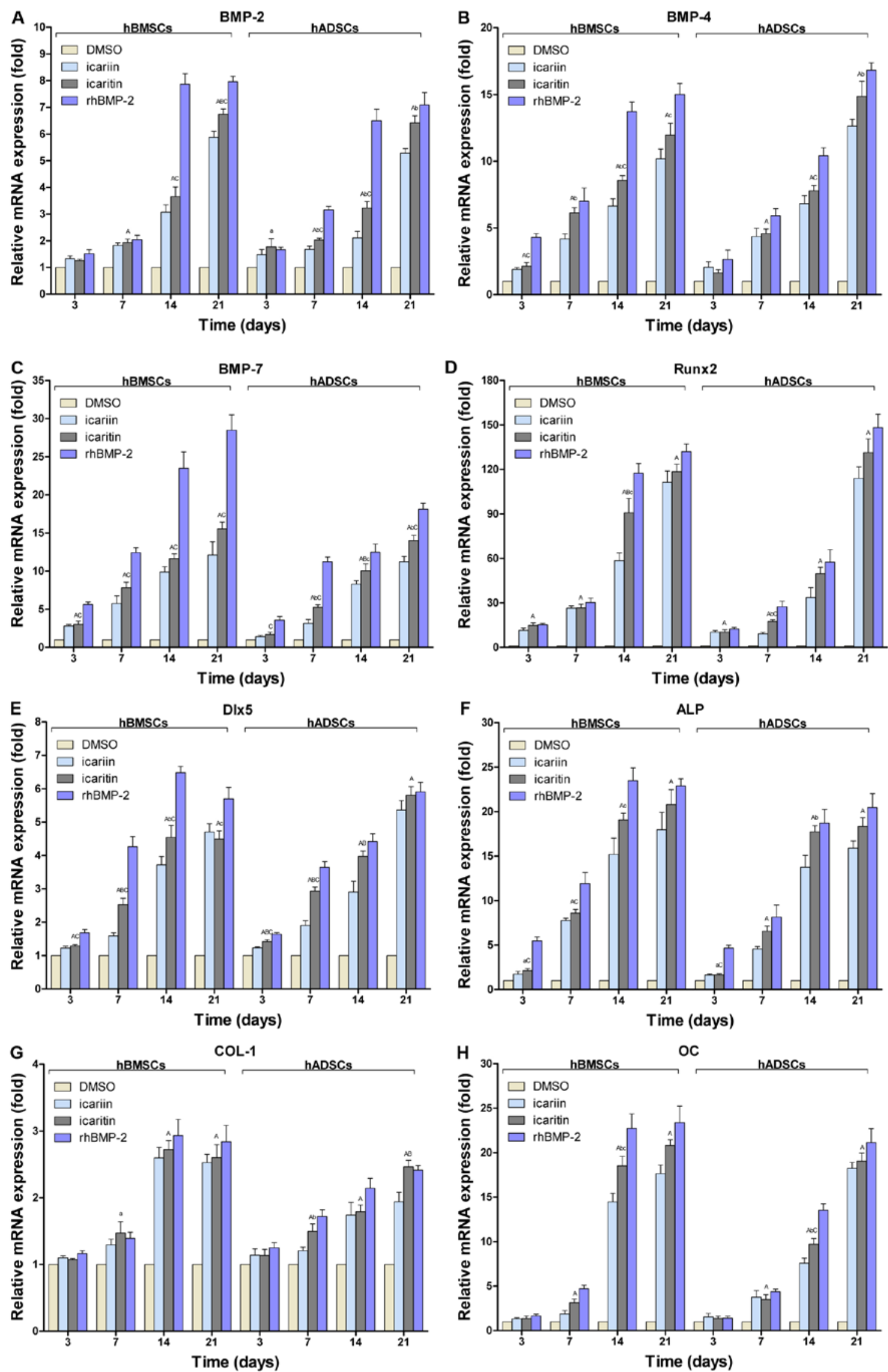

Figure 3. Effects of icaritin on the mRNA expression of osteogenic marker genes in hBMSCs and hADSCs. hBMSCs and hADSCs were treated with DMSO control, $10^{-6} \mathrm{M}$ icariin, $10^{-6} \mathrm{M}$ icaritin or $100 \mathrm{ng} / \mathrm{ml}$ rhBMP-2, and gene expression was determined by RT-qPCR. Data are the means \pm SD ( $=6$, ${ }^{A} \mathrm{P}<0.01 \mathrm{vs.} \mathrm{control,}$ ${ }^{\mathrm{a}} \mathrm{P}<0.05$ vs. control; ${ }^{\mathrm{B}} \mathrm{P}<0.01$ vs. icariin, ${ }^{\mathrm{b}} \mathrm{P}<0.05$ vs. icariin; ${ }^{\mathrm{C}} \mathrm{P}<0.01$ vs. rhBMP-2, ${ }^{\mathrm{c}} \mathrm{P}<0.05$ vs. rhBMP-2). hBMSCs, human bone marrow-derived mesenchymal stem cells; rhBMPs, recombinant human bone morphogenetic proteins; hADSCs, human adipose tissue-derived stem cells; DMSO, dimethyl sulfoxide.; n, number of experiments 


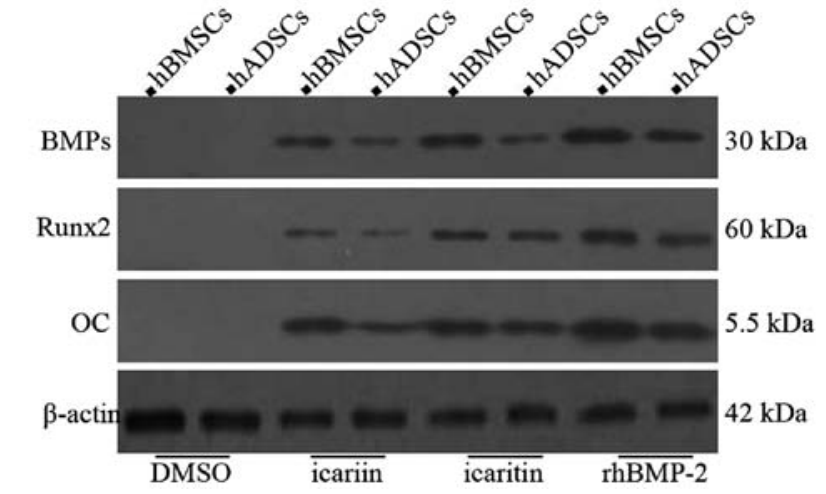

Figure 4. Effects of icaritin on osteogenic protein expression in hBMSCs and hADSCs. Cells were exposed to DMSO control, $10^{-6} \mathrm{M}$ icariin, $10^{-6} \mathrm{M}$ icaritin or $100 \mathrm{ng} / \mathrm{ml}$ rhBMP-2 for 14 days. The expression levels of BMPs, Runx 2 and osteocalcin (OC) were determined by western bot analysis, using $\beta$-actin as an internal control. Protein size indicated in kDa. hBMSCs, human bone marrow-derived mesenchymal stem cells; hADSCs, human adipose tissue-derived stem cells; DMSO, dimethyl sulfoxide.

can also promote osteogenic differentiation of bone marrow stromal cells (35) and play a bone protective role $(23,36,37)$. However, whether icaritin can be used as a bone induction factor, has not been systematically reported.

Multipotential stem cells have an excellent ability to differentiate into osteogenic, fibroblastic, myogenic and adipogenic cells. In this study, BMSCs and ADSCs were chosen as target cells for investigating the osteoinductive properties of icaritin. In the beginning, we inspected the cytotoxicity of icaritin. The results showed that icaritin did not affect the cell viability of hBMSCs and hADSCs at a suitable concentration range from $10^{-8} \mathrm{M}$ to $10^{-5} \mathrm{M}$; however, cytotoxicity was confirmed at concentrations above $10^{-5} \mathrm{M}$, which limits its use. Researchers have found that the effective drug concentration ranges mainly from $10^{-10} \mathrm{M}$ to $10^{-5} \mathrm{M}(17,19,24,33,34,38)$, which is consistent with the results of the cytotoxicity experiment in this study.

It is well known that adult stem cells play an important role in the bone regeneration process, which involves a complex cascade of events including the recruitment and proliferation of osteoprogenitors, cell differentiation, osteoid formation, and ultimately mineralization (39). There are significant changes in the expression of functional markers during the differentiation of osteoprogenitors into osteoblasts in different phases. ALP is an early marker for osteogenic differentiation (40). We found that icaritin, especially at $10^{-6} \mathrm{M}$ concentration, increased ALP activity in the hBMSCs and hADSCs at day 3, and then ALP activity increased significantly with time. OC is a bone-specific extracellular matrix protein expressed by mature osteoblasts during later stages of differentiation (40). This study showed that icaritin increased OC activity in hBMSCs and hADSCs at day 14 and 21 , and $10^{-6} \mathrm{M}$ was observed to be the optimum treatment concentration. In addition, the presence of more ALP-positive stained cells and calcium nodules compared to that in the DMSO control group observed by cytologic dyeing indicated that more cells in the well committed to differentiation into an osteoblastic lineage after treatment with $10^{-8}-10^{-5} \mathrm{M}$ icaritin.

Osteogenic differentiation of cells employs a complex cascade, which is regulated by osteoblast-specific genes and proteins. BMPs play an important role in regulating these processes. They activate Smads and p38 MAPK via the Smad and MAPK signaling pathways by combining with specific serine/threonine kinase receptors on the cell membrane, and then these signals are transferred into the nucleus. The promoters of bone matrix downstream genes (e.g., ALP, OC, BSP) link to the activating transcription factors of Cbfal, Osx, and Dlx5, and this induces the transcription of osteogenic genes (41). In addition, BMPs can also interact with other growth factors to modify and promote bone formation. Dlx5, the earliest target of BMP-activated Smads, is an osteoblast-specific and BMP signaling-specific transcription factor. It crucially coordinates Runx2, a downstream osteogenic master gene, and Osx expression which in turn work sequentially and/or work together to induce the expression of bone marker genes (42). The core-binding factor $\alpha-1$ (Cbfa-1/Runx2) is known as the master switch that initiates osteoblast differentiation (39). Most of the well-established bone markers, such as ALP, collagen I, bone sialoprotein, osteopontin, and $\mathrm{OC}$ are known target genes of Cbfa1. ALP, OC and COL-1 are specific proteins involved in the process of osteoblast differentiation from immature osteoblasts to mature osteoblasts, and the mRNA expression of these proteins can reflect the maturity of osteoblasts $(43,44)$. Although the osteogenesis regulatory mechanism of icaritin in mesenchymal stem cells has not been elucidated, the osteogenesis regulatory mechanism of icariin, which has a similar molecular structure, has been widely studied. There is clear evidence that the osteogenic effect of icariin requires the activation of BMP signaling and Runx 2 transcriptional activity. The addition of noggin, a BMP antagonist, into the culture medium decreased the icariin-induced expression levels of OC and BSP mRNAs in MC3T3-E1 cells (45). Further studies demonstrated that the total flavonoids present in Epimedii increased Cbfal expression in the bone of ovariectomized rats (46). Furthermore, icariin induced the mRNA expression of BMP-2 and BMP-4 in osteoblasts and low doses of icariin significantly elevated Osx mRNA expression $(45,47,48)$. In addition, it enhanced ALP, OPN, and type 1 collagen mRNA expression in a dose-dependent manner in vitro (49). Here, the mRNA levels of BMP-2, -4 and -7 were markedly enhanced after treatment with icaritin at different time points. This data combined with the results of Dlx5, Runx2, ALP, OC and COL-1 mRNA levels suggest that icaritin directly or indirectly activates BMP signaling, induces the expression of transcription factor genes, upregulates bone matrix genes, and terminally leads to osteogenic differentiation in hBMSCs and hADSCs. In terms of the levels of genes expressed, our data showed that icaritin was more potent than icariin in promoting osteogenic differentiation.

Since gene expression analysis is an indirect method for assessing the relative abundance of protein expression, we selected three representative proteins, Cbfa1, BMPs and OC, to assess the osteogenic differentiation effect of icaritin. Data from the western blot analysis demonstrated that icaritin directly stimulated the synthesis of BMPs, Cbfa1 and OC. Thus, we suggest that the mechanism underlying the induction of osteogenic differentiation by icaritin is possibly through the direct stimulation of BMP production.

As the structure is similar to mammalian estrogens, icariin and its derivatives have been considered as phytoestrogens 
and are believed to possess estrogenic-like activities $(18,50)$. Estrogen can improve osteoblast proliferation, differentiation, and synthesis of metal matrix protein by combining with an estrogen receptor on the surface of cells. However, whether icaritin promotes the osteogenetic differentiation of hBMSCs and hADSCs via estrogen-like effects warrants further research.

In conclusion, although icaritin showed a lower efficacy of osteogenic differentiation than rhBMP-2, icaritin has the advantages of low cost, is easily available as a raw material and requires simple extraction technology. Additionally, icaritin has the following properties: high melting point $\left(>250^{\circ} \mathrm{C}\right)$, chemical stability, easy sterilization and storage. Therefore, icaritin has potential to serve as an osteoinductive agent in bone regenerative medicine.

\section{Acknowledgements}

This study was funded by the National Natural Science Foundation of China (grant no. 81303107), the China Postdoctoral Science Foundation (no. 2014M552272), the Natural Science Foundation of Guangdong Province (grant no. S2012040006295) and the Medical Science Research Foundation of Guangdong Province (grant nos. A2012499 and A2014499).

\section{References}

1. Ding S, Li L, Liu X, Yang G, Zhou G and Zhou S: A nano-micro alternating multilayer scaffold loading with rBMSCs and BMP-2 for bone tissue engineering. Colloids Surf B Biointerfaces 133: 286-295, 2015.

2. Rahman CV, Ben-David D, Dhillon A, Kuhn G, Gould TW, Müller R, Rose FR, Shakesheff KM and Livne E: Controlled release of BMP-2 from a sintered polymer scaffold enhances bone repair in a mouse calvarial defect model. J Tissue Eng Regen Med 8: 59-66, 2014.

3. Su J, Xu H, Sun J, Gong X and Zhao H: Dual delivery of BMP-2 and bFGF from a new nano-composite scaffold, loaded with vascular stents for large-size mandibular defect regeneration. Int J Mol Sci 14: 12714-12728, 2013

4. Wong E, Sangadala S, Boden SD, Yoshioka K, Hutton WC Oliver $\mathrm{C}$ and Titus L: A novel low-molecular-weight compound enhances ectopic bone formation and fracture repair. J Bone Joint Surg Am 95: 454-461, 2013.

5. Hu ZM, Peel SA, Sandor GK and Clokie CM: The osteoinductive activity of bone morphogenetic protein (BMP) purified by repeated extracts of bovine bone. Growth Factors 22: 29-33, 2004

6. Okada M, Sangadala S, Liu Y, Yoshida M, Reddy BV, Titus L and Boden SD: Development and optimization of a cell-based assay for the selection of synthetic compounds that potentiate bone morphogenetic protein-2 activity. Cell Biochem Funct 27: 526-534, 2009

7. Fan JJ, Cao LG, Wu T, Wang DX, Jin D, Jiang S, Zhang ZY, $\mathrm{Bi} \mathrm{L}$ and Pei GX: The dose-effect of icariin on the proliferation and osteogenic differentiation of human bone mesenchymal stem cells. Molecules 16: 10123-10133, 2011.

8. Franceschi RT, Yang S, Rutherford RB, Krebsbach PH, Zhao M and Wang D: Gene therapy approaches for bone regeneration. Cells Tissues Organs 176: 95-108, 2004.

9. Wutzl A, Brozek W, Lernbass I, Rauner M, Hofbauer G, Schopper C, Watzinger F, Peterlik M and Pietschmann P: Bone morphogenetic proteins 5 and 6 stimulate osteoclast generation. J Biomed Mater Res A 77: 75-83, 2006.

10. Kim RY, Oh JH, Lee BS, Seo YK, Hwang SJ and Kim IS: The effect of dose on rhBMP-2 signaling, delivered via collagen sponge, on osteoclast activation and in vivo bone resorption. Biomaterials 35: 1869-1881, 2014.

11. Zhang J, Zhao Y, Hou X, Chen B, Xiao Z, Han J, Shi C, Liu J, Miao Q and Dai J: The inhibition effects of insulin on BMP2-induced muscle heterotopic ossification. Biomaterials 35: 9322-9331, 2014.
12. Wu T, Xu JC and Nan KH: Icariin enhances proliferation and osteogenic differentiation of goat bone marrow mesenchymal stem cells. J Clin Rehabilitative Tissue Eng Res 13: 3725-3729, 2009.

13. Fan J, Bi L, Wu T, Cao L, Wang D, Nan K, Chen J, Jin D, Jiang S and Pei G: A combined chitosan/nano-size hydroxyapatite system for the controlled release of icariin. J Mater Sci Mater Med 23: 399-407, 2012.

14. Wu T, Nan KH, Chen JD, Jin D, Jiang S, Zhao PR, Xu JC, Du H, Zhang XQ, Li JW and Pei GX: A new bone repair scaffold combined with chitosan/hydroxyapatite and sustained releasing icariin. Chin Sci Bull 54: 2953-2961, 2009.

15. Ye LH, Xiao BX, Cao FR, Zheng Y, Pan RL and Chang Q: Identification of icaritin metabolites in rats by LC-MS/MS. Chin Herb Med 7: 296-302, 2015.

16. Yao ZH, Liu MY, Dai Y, Zhang Y, Qin ZF, Tu FJ and Yao XS: Metabolism of Epimedium-derived flavonoid glycosides in intestinal flora of rabbits and its inhibition by gluconolactone. Chin J Nat Med 9: 461-465, 2011.

17. Wang ZQ and Lou YJ: Proliferation-stimulating effects of icaritin and desmethylicaritin in MCF-7 cells. Eur J Pharmacol 504: $147-153,2004$.

18. Kang HK, Choi YH, Kwon H, Lee SB, Kim DH, Sung CK, Park YI and Dong MS: Estrogenic/antiestrogenic activities of a Epimedium koreanum extract and its major components: In vitro and in vivo studies. Food Chem Toxicol 50: 2751-2759, 2012.

19. Ye HY and Lou YJ: Estrogenic effects of two derivatives of icariin on human breast cancer MCF-7 cells. Phytomedicine 12: 735-741, 2005.

20. Wong SP, Shen P, Lee L, Li J and Yong EL: Pharmacokinetics of prenylflavonoids and correlations with the dynamics of estrogen action in sera following ingestion of a standardized Epimedium extract. J Pharm Biomed Anal 50: 216-223, 2009.

21. Liu YQ, Yang QX, Cheng MC and Xiao HB: Synergistic inhibitory effect of Icariside II with Icaritin from Herba Epimedii on pre-osteoclastic RAW264.7 cell growth. Phytomedicine 21: 1633-1637, 2014.

22. Yao D, Wang X, Xie X, Zhang G and Qin L: Icaritin promotes osteogenic differentiation while inhibits osteoclastic differentiation in vitro. Can Nurse 76: 510-519, 1980.

23. Long J,Zhou Q, Li D, Wang X, Cao H and Qin L: Phytoestrogenic molecule icaritin prevents OVX-induced osteoporosis in mice. J Orthop Transl 2: 224-225, 2014.

24. Sheng H, Rui XF, Sheng CJ, Li WJ, Cheng XY, Jhummon NP, Yu YC, Qu S, Zhang G and Qin L: A novel semisynthetic molecule icaritin stimulates osteogenic differentiation and inhibits adipogenesis of mesenchymal stem cells. Int J Med Sci 10: 782-789, 2013.

25. Qin L, Yao D, Zheng L, Liu WC, Liu Z, Lei M, Huang L, Xie X, Wang X, Chen Y, et al: Phytomolecule icaritin incorporated PLGA/TCP scaffold for steroid-associated osteonecrosis: Proof-of-concept for prevention of hipjoint collapse in bipedalemus and mechanistic study in quadrupedal rabbits. Biomaterials 59: 125-143, 2015.

26. Chen SH, Wang XL, Xie XH, Zheng LZ, Yao D, Wang DP, Leng Y, Zhang G and Qin L: Comparative study of osteogenic potential of a composite scaffold incorporating either endogenous bone morphogenetic protein-2 or exogenous phytomolecule icaritin: An in vitro efficacy study. Acta Biomater 8: 3128-3137, 2012

27. Zhang G, Qin L, Sheng H, Wang XL, Wang YX, Yeung DK, Griffith JF, Yao XS, Xie XH, Li ZR, et al: A novel semisynthesized small molecule icaritin reduces incidence of steroid-associated osteonecrosis with inhibition of both thrombosis and lipid-deposition in a dose-dependent manner. Bone 44: 345-356, 2009.

28. Wang XL, Xie XH, Zhang G, Chen SH, Yao D and Qin L: An innovative porous scaffold incorporating exogenous phytoestrogenic molecule icaritin for enhancement of bone defect repair. Bone 47: S418, 2010.

29. Kyllönen L, D'Este M, Alini M and Eglin D: Local drug delivery for enhancing fracture healing in osteoporotic bone. Acta Biomater 11: 412-434, 2015.

30. Zhang X, Liu T, Huang Y, Wismeijer D and Liu Y: Icariin: Does it have an osteoinductive potential for bone tissue engineering? Phytother Res 28: 498-509, 2014.

31. Zhao J, Ohba S, Komiyama Y, Shinkai M, Chung UI and Nagamune T: Icariin: A potential osteoinductive compound for bone tissue engineering. Tissue Eng Part A 16: 233-243, 2010.

32. Zhang SQ: Ultra-high performance liquid chromatographytandem mass spectrometry for the quantification of icaritin in mouse bone. J Chromatogr B Analyt Technol Biomed Life Sci 978-979: 24-28, 2015. 
33. Huang J, Yuan L, Wang X, Zhang TL and Wang K: Icaritin and its glycosides enhance osteoblastic, but suppress osteoclastic, differentiation and activity in vitro. Life Sci 81: 832-840, 2007.

34. Zhu RQ, Li ZZ, Zhou J, Han GQ, Cheng GZ and Zhai YK Estrogenic activities of icariin and its possible metabolites in vitro. Acta Chin Med Pharmacol 40: 15-20, 2012.

35. Zhai YK, Li ZZ, Chen KM, Zhang N, Cheng GZ and Zhu RQ: Comparative study on the effects of the osteogenic differentiation of rat bone marrow stromal cells by icaritin and dehydrated icaritin. J Chin Pharm Sci 46: 837-842, 2011.

36. Guo B, Zhang G, He Y, Wang X, Xie X, Hung L and Qin L: Icaritin with dual action prevents ovariectomy-induced osteoporosis in mice: Beneficial effect on bone and muscle via estrogen-receptor-dependent and independent pathway, respectively. Bone 47: S359, 2010.

37. He Y,Zhang G, Wang X, Guo B, Xie X, Hung L and Qin L: Icaritin, as secondary metabolites of bone-strengthening herb epimedium, dose-dependently prevents estrogen-depletion-induced bone loss in ovariectomized rats. Bone 47: S404-S405, 2010.

38. Yao D, Xie XH, Wang XL, Wan C, Lee YW, Chen SH, Pei DQ, Wang YX, Li G and Qin L: Icaritin, an exogenous phytomolecule, enhances osteogenesis but not angiogenesis - an in vitro efficacy study. PLoS One 7: e41264, 2012.

39. Aubin JE: Regulation of osteoblast formation and function. Rev Endocr Metab Disord 2: 81-94, 2001.

40. Wagner ER, Luther G, Zhu G, Luo Q, Shi Q, Kim SH, Gao JL, Huang E, Gao Y, Yang K, et al: Defective osteogenic differentiation in the development of osteosarcoma. Sarcoma 2011: 325238 , 2011.

41. Ryoo HM, Lee MH and Kim YJ: Critical molecular switches involved in BMP-2-induced osteogenic differentiation of mesenchymal cells. Gene 366: 51-57, 2006.

42. Ulsamer A, Ortuño MJ, Ruiz S, Susperregui AR, Osses N, Rosa JL and Ventura F: BMP-2 induces Osterix expression through up-regulation of Dlx5 and its phosphorylation by $\mathrm{p} 38$. J Biol Chem 283: 3816-3826, 2008.
43. Wang RY and Jing YI: Signaling mechanism of osteoblastic differentiation of bone morphogenetic proteins Chin. Bull Life Sci 17: 34-39, 2005.

44. Choi MH, Noh WC, Park JW, Lee JM and Suh JY: Gene expression pattern during osteogenic differentiation of human periodontal ligament cells in vitro. J Periodontal Implant Sci 41: $167-175,2011$.

45. Zhao J, Ohba S, Shinkai M, Chung UI and Nagamune T: Icariin induces osteogenic differentiation in vitro in a BMP- and Runx2-dependent manner. Biochem Biophys Res Commun 369: 444-448, 2008

46. Qian G, Zhang X, Lu L, Wu X, Li S and Meng J: Regulation of Cbfa1 expression by total flavonoids of Herba epimedii. Endocr J 53: 87-94, 2006

47. Yin XX, Chen ZQ, Liu ZJ, Ma QJ and Dang GT: Icariine stimulates proliferation and differentiation of human osteoblasts by increasing production of bone morphogenetic protein 2 . Chin Med J (Engl) 120: 204-210, 2007.

48. He W, Li Z, Yi B, Liang C, Wang X, Li Y and Wang X: Icariin upregulated $\mathrm{Cbfa} 1$, bone morphogenetic protein 2 and bone morphogenetic protein 4 mRNA expression of rat osteoblast. Int J Oral Max Surg 38: 459, 2009.

49. Xiao Q, Chen A and Guo F: Effects of Icariin on expression of OPN mRNA and type I collagen in rat osteoblasts in vitro. J Huazhong Univ Sci Technolog Med Sci 25: 690-692, 2005.

50. Shen P, Guo BL, Gong Y, Hong DY, Hong Y and Yong EL: Taxonomic, genetic, chemical and estrogenic characteristics of Epimedium species. Phytochemistry 68: 1448-1458, 2007. 